\title{
Parametric design of mechanical dither with bimorph piezoelectric actuator for ring laser gyroscope
}

\author{
Xudong $\mathrm{Yu}^{*}$ and Xingwu Long \\ College of Optoelectronic Science and Engineering, National University of Defense Technology, \\ Changsha, Hunan, China
}

\begin{abstract}
This paper presents a mathematical model and a finite element model to investigate the model parameters of mechanical dither in a ring laser gyroscope in which the bimorph piezoelectric actuator are employed as driving mechanisms to avoid the problem of so-called lock-in effects. The validity and efficiency of the mathematical model and finite element model are conformed by comparing with the sinusoidal experimental results, and the errors are $3.14 \%$ and $1.79 \%$ respectively. Moreover, the parametric design for the geometric dimensions of mechanical dither and the bimorph piezoelectric actuators is easily achieved through finite element model. It is shown that careful selection of the structural parameters allows the resonant frequency to be suitable. These results are important for the mechanical dither design and improvement of high accuracy ring laser gyroscopes and inertial navigation system.
\end{abstract}

Keywords: Dithered ring laser gyroscope, mechanical dither, bimorph piezoelectric actuator, finite element analysis

\section{Introduction}

The ring laser gyroscope (RLG), based on the principle of Sagnac effect, is a kind of core sensor in inertial systems which has been widely used in numerous cutting-edge military fields [1,2]. Due to its widely dynamic range and bandwidth, high accuracy and reliability, and relatively low acquisition costs, the RLG has become extremely attractive for navigation systems [3,4].

Of the various error sources in RLG, the most limiting one is the mode-locking or lock-in phenomenon. Such problems can be alleviated by mounting the laser block on a mechanical dither which oscillate the block about the rotation axis and suppress the dead band [5]. A bimorph piezoelectric actuator is bonded by epoxy on each side of the spoke of the mechanical dither concurrently for dither-rate sensing and dither-motion actuation. The mechanical dither affects the structural resonant frequency, peak angular amplitude, peak dither rate and mechanical properties. In an inertial navigation system, three gyros mounted orthogonally dither at three different resonant frequencies to avoid mechanical coupling and dithering beating [6,7]. Thus a precise design and selection of the resonant frequency for mechanical dither is required. In order to optimize the performance of the dithered RLG, it is necessary

\footnotetext{
${ }^{*}$ Corresponding author: Xudong Yu, College of Optoelectronic Science and Engineering, National University of Defense Technology, Changsha 410073, Hunan, China. E-mail: wind0909@163.com.
}

1383-5416/15/\$35.00 (C) 2015 - IOS Press and the authors. All rights reserved

This article is published online with Open Access and distributed under the terms of the Creative Commons Attribution NonCommercial License. 


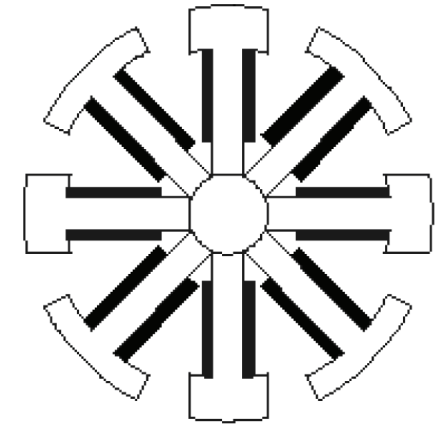

Fig. 1. Schematic diagram of mechanical dither.

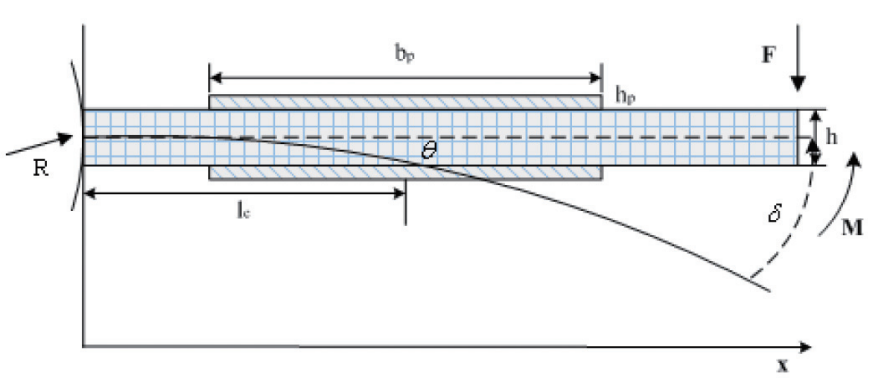

Fig. 2. Geometry and force analysis of the spoke.

to understand the resonant frequency response as a function of geometry and material for mechanical dither with bimorph piezoelectric actuators.

The dither mechanism design was firstly explained by Shackleton [8]. Lee. presented the performances of dither mechanism theoretically on the basis of the loading condition and angular characteristics due to piezoelectric ceramic deformation [9-11]. A numerical modeling of the piezoelectric actuator included piezoelectric rigidity effect of the actuator to predict the resonant frequency of the mechanical dither. Yang adopted a finite element method to investigate the resonant frequency at different piezoelectric element and bonding layer thicknesses [5]. The layer configurations and flexural stiffness of multilayered materials was established based on plate/shell theory to obtain the angular displacements of mechanical dither [12]. The author presented an optimal structural design of mechanical dither using finite element analysis [13].

The above studies have all had some success in analytical modeling the mechanical dither with bimorph piezoelectric actuators. However, the resonant frequency and peak dither rate of dithering mode are mainly focused. Many issues such as several geometric parameters of the spoke and the bimorph piezoelectric actuators have not been described fully. To this end, this paper focuses on the development of a finite element method and experiment to investigate the geometric and structural parameters of the mechanical dither with bimorph piezoelectric actuators.

\section{Mathematical models}

The mechanical dither includes a central hub and a plurality of spokes extending therefrom as shown in Fig. 1. One half of the spokes are connected to a rim which is coaxial with the hub and attached to the resonant cavity, and the other half are connected to a support base which is fixed to the mounting case. The bimorph piezoelectric actuators are bond by epoxy on each side of the spoke. The mechanical dither is operated at the fundamental structural frequency which is the first resonant frequency of the dithered RLG.

When the bimorph piezoelectric actuators are bonded to the spokes, a generalized form of Hamilton's principle for mechanical dither can be formulated [14].

$$
\int_{t_{1}}^{t_{2}} \delta(T-U+W) d t=0
$$


Where $T$ is the total kinetic energy, $U$ is the total potential energy and $W$ is the external work applied to the system.

Using the piezoelectric constitutive equation and the Hamilton's principle, the equation of the mechanical dither can be obtained. According to the Euler-Bernoulli beam assumptions, the generalized equation of motion for mechanical dither can be derived as follow.

$$
\begin{aligned}
M \ddot{r}+K r-\Theta v & =\sum_{i=1} \varphi_{i}^{t} f_{i} \\
\Theta^{t} r+C_{P} v & =-q
\end{aligned}
$$

where $r$ is the generalized displacement coordinate vector and $v$ is the generalized electrical coordinate vector, $M, K, \Theta, C_{P}$ are the mass matrix, stiffness matrix, electromechanical coupling matrix and the capacitance matrix respectively.

A basic geometry of the spoke which is sandwiched between the bimorph piezoelectric actuators is shown in Fig. 2. The modeling of this structure neglects shear effects and ignores residual stress induced curvature. In addition, the beam thickness is much less than the piezoelectric-induced curvature, so the second-order effects such as electrostriction can be ignored. At equilibrium, both axial forces and moments must sum to zero for any cross section of the spoke $[15,16]$.

$$
\begin{aligned}
\sum_{i=1}^{m} F_{i} & =0 \\
\sum_{i=1}^{m} M_{i} & =F_{1}\left(\frac{h_{1}}{2}\right)+F_{2}\left(t_{1}+\frac{h_{2}}{2}\right)+\ldots+F_{m}\left(\sum_{i=1}^{m-1} t_{i}+\frac{h_{m}}{2}\right)=M_{t}
\end{aligned}
$$

where $F_{i}, M_{i} h_{i}$ are axial force, moment and thickness of the $i_{t h}$ layer. Under the same bending moment, the radius of curvature is the same under deformation.

$$
\frac{1}{r}=\frac{1}{\sum_{i=1}^{m} E_{i} I_{i}} M_{t}=\frac{1}{\sum_{i=1}^{m} E_{i} I_{i}}\left(F_{1}\left(\frac{h_{1}}{2}\right)+F_{2}\left(t_{1}+\frac{h_{2}}{2}\right)+\ldots+F_{m}\left(\sum_{i=1}^{m-1} t_{i}+\frac{h_{m}}{2}\right)\right)
$$

The total strain at the surface of each layer is the sum of the strains caused by the piezoelectric effect, the axial force, and the bending [17].

$$
d_{31} E_{i}^{p}+\frac{F_{i}}{A_{i} E_{i}}-\frac{h_{i}}{2 r}=d_{31} E_{i+1}^{p}+\frac{F_{i+1}}{A_{i+1} E_{i+1}}+\frac{h_{i+1}}{2 r}
$$

where $d_{31}$ is transverse piezoelectric coupling coefficient, $E_{i}^{p}$ is the electric field across the $i_{\text {th }}$ layer. From Eqs (4)-(7), the deflection and angle of the spoke with bimorph piezoelectric actuators can be calculated as

$$
\delta=\frac{x(l)^{2}}{2 r}, \quad \theta=\frac{x(l)^{2}}{2 r l}
$$

The torsional stiffness of the spoke is

$$
K_{\theta}=\frac{M_{t}}{\theta}
$$


Table 1

Material properties of the dithered RLG

\begin{tabular}{llccc}
\hline Component & Material & Density $\left(\mathrm{kg} / \mathrm{m}^{3}\right)$ & Young's modulus $(\mathrm{GPa})$ & Poisson's ratios \\
\hline Glass-cavity & Crystallite glass & 2530 & 90 & 0.243 \\
Mechanical dither & Super invar & 8100 & 141 & 0.35 \\
bimorph piezoelectric actuator & PZT-5H & 7800 & 70 & 0.31 \\
Anode & Copper & 8960 & 129 & 0.343 \\
Cathode & Aluminum & 2800 & 72 & 0.31 \\
\hline
\end{tabular}

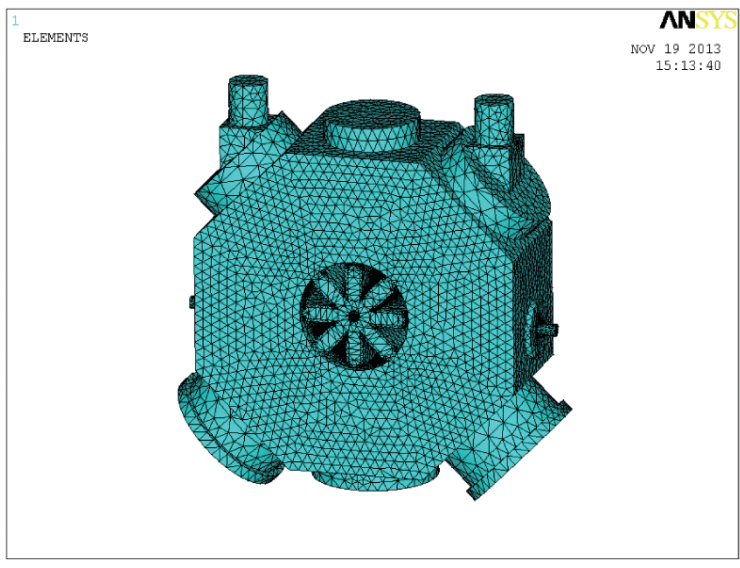

Fig. 3. Finite element modal of dithered RLG.

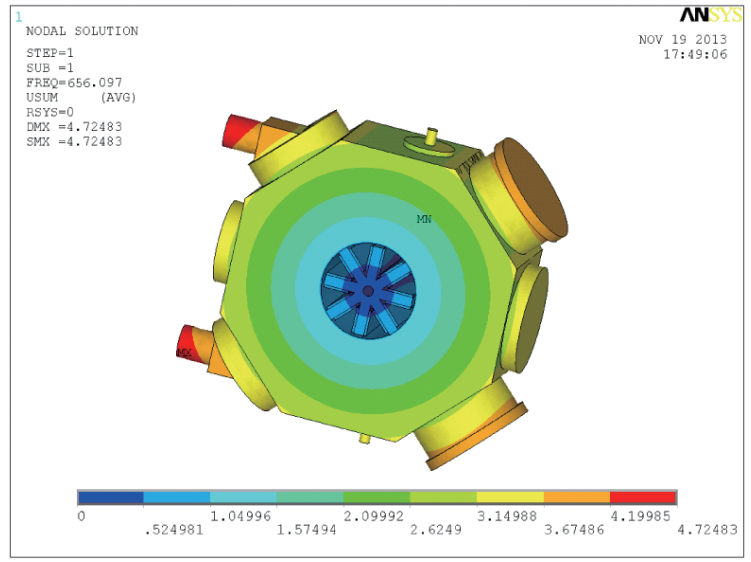

Fig. 4. Modal analysis of dithered RLG.

According to Eq. (2), the resonant frequency for the mechanical dither with bimorph piezoelectric actuators may be given by [18]:

$$
\omega=\sqrt{\frac{n K_{\theta}}{J_{\text {sys }}}}
$$

where $n$ is the number of the spokes, $K_{\theta}$ is the torsional stiffness of the mechanical dither from Eq. (9), $J_{s y s}$ is the mass moment of inertia of the dithered RLG from mass matrix $M$.

From Eq. (10), we can obtain the resonant frequency is strictly related to the geometrical and structural characteristics of the mechanical dither, particularly to the cross-sectional moment of inertial of the spoke and bimorph piezoelectric actuators. The influence of geometric variation of the mechanical dither with bimorph piezoelectric actuators can not depicted clearly from this numerical equation. Therefore, a more accurate method to investigate the structural characteristics of the mechanical dither should be introduced.

\section{Simulation and experiments}

The ANSYS 12.0 finite element program is used for modal analysis of the mechanical dither with bimorph piezoelectric actuators. A B-type mechanical dither is modeling and the finite element model is shown in Fig. 3. The material properties of the RLG are given in Table 1. Block Lanczos Method is conducted to solve the model and the result of the first resonant frequency and mode shape is shown in 
Table 2

Comparison of resonant frequencies for mechanical dither with bimorph piezoelectric actuators

\begin{tabular}{cccccc}
\hline Type & $\begin{array}{c}\text { Experiment } \\
(\mathrm{Hz})\end{array}$ & $\begin{array}{c}\text { Mathematical } \\
\text { model }(\mathrm{Hz})\end{array}$ & Error & $\begin{array}{c}\text { Finite element } \\
\text { method }(\mathrm{Hz})\end{array}$ & Error \\
\hline A-type & 736 & 749 & $1.77 \%$ & 732 & $0.54 \%$ \\
B-type & 668 & 687 & $3.14 \%$ & 656 & $1.79 \%$ \\
C-type & 616 & 626 & $1.62 \%$ & 615 & $0.16 \%$ \\
\hline
\end{tabular}

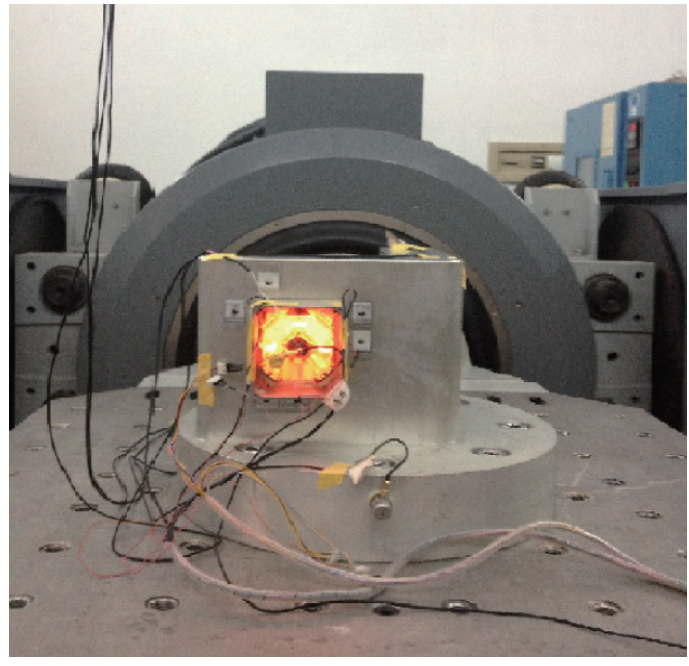

Fig. 5. Sinusoidal vibration apparatus for the RLG.

Fig. 4. The first mode is a dithering mode as the glass cavity rotates back and forth around the central axis at $656 \mathrm{~Hz}$ and the structural deformations mainly generates from the mechanical dither.

A sinusoidal vibration experiment is performed to compare the mathematical model and finite element model of the mechanical dither with experimental results. An accelerometer mounted on the dithered RLG is used to measure the acceleration response of the structure. Experimental apparatus is shown in Fig. 5.

Using the mathematical equations, the resonant frequencies of mechanical dither are compared with finite element method and experiment. In Table 2, the results of the mathematical model and finite element method show that the maximum relative errors to experimental results are within $3.14 \%$ and $1.79 \%$ respectively. The finite element method is more accurate than the mathematical equations. The sinusoidal vibration experiment validated the results of the mathematical model and finite element method which can be the efficient theoretical instruction for designing mechanical dither with bimorph piezoelectric actuators. A modal analysis which ignored the bimorph piezoelectric actuators is also conducted and the resonant frequency is $619 \mathrm{~Hz}$ for B-type mechanical dither. The difference is as much as $49 \mathrm{~Hz}$. This verification typifies that the inertia and stiffness induced by the bimorph piezoelectric actuators can be of significant importance to resonant frequency calculation. Therefore, carefully selection and geometric design for mechanical dither and bimorph piezoelectric actuators are important for dithered RLG.

\section{Results and discussion}

Based on the finite element method in Section 3, the influence of geometric parameters on the mechanical dither with bimorph piezoelectric actuators are given as below. The geometric parameters of interest (shown in Fig. 2), which include those of the spoke (thickness $h$ and radius $R$ of central hole) and the bimorph piezoelectric actuators (thickness $h_{p}$, length $l_{p}$, width $b_{p}$, location $l_{c}$ ) are examined respectively. Analyses on the finite element method are performed varying all of the aforementioned parameters for modal analysis. 


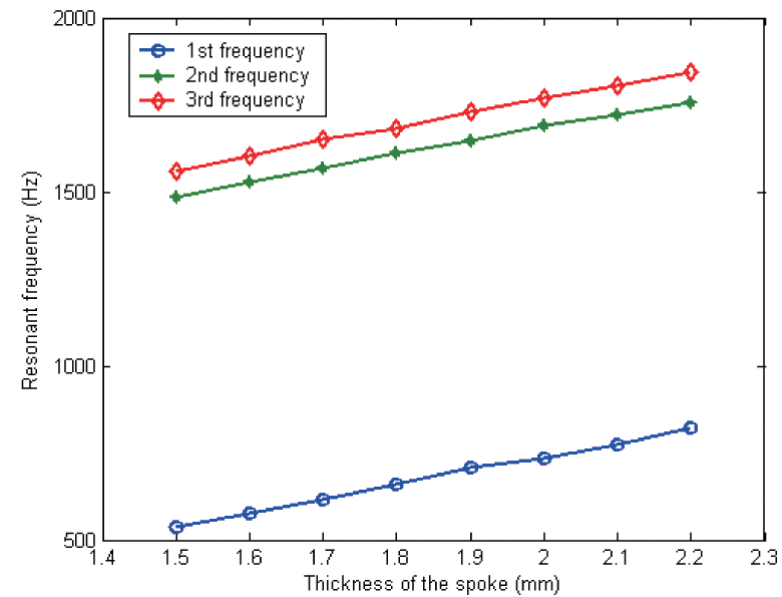

Fig. 6. The first three order frequencies of the dithered RLG at different spoke thicknesses.

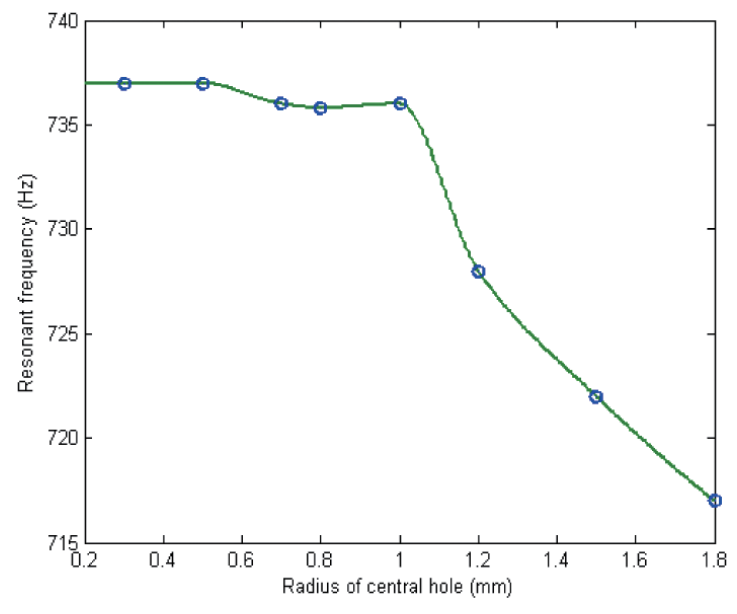

Fig. 7. The relationship between the resonant frequency and radius of central hole.

\subsection{Effect of the geometrical dimensions of the spoke on resonant frequency}

In this case, the geometrical parameters and material of the bimorph piezoelectric actuators are fixed. The resonant frequency is obtained while varying the thickness $h$ of the spoke from $1.5 \mathrm{~mm}$ to $2.2 \mathrm{~mm}$. The second and third resonant frequencies are also plotted in Fig. 6 as a function of thickness $h$. The first three order frequencies of the RLG structure are in almost linear relation to the thickness changes which are absolutely coincident with reference [5]. A small deviation of $0.1 \mathrm{~mm}$ in the spoke thickness can lead to a shift of about $40 \mathrm{~Hz}$ in those frequencies. The physical explanation to this phenomenon is that the structure deformations on these frequencies are mainly on the spoke, when the spoke thickness is increased, the equivalent rigidity is increased, and therefore, the first three order frequencies are increased obviously.

The effect of the radius $R$ of central hole on the performance of the dithered RLG is analyzed when the thickness $h$ of the spoke is fixed as $2.0 \mathrm{~mm}$. In the Fig. 7, it is found that the difference on resonant frequency is very small when the radius is less than $1 \mathrm{~mm}$. An interesting phenomenon is found when the radius is bigger than $1 \mathrm{~mm}$ and increased by $0.2 \mathrm{~mm}$, the resonant frequency is decreased by $6 \mathrm{~Hz}$.

\subsection{Effect of the geometrical dimensions of the bimorph piezoelectric actuators on resonant frequency}

When the geometrical dimensions of the spoke are fixed, the effect of the thickness $h_{p}$ of the bimorph piezoelectric actuators is also analyzed. Figure 8 is the simulated relationship between the resonant frequency and the thickness $h_{p}$ of the bimorph piezoelectric actuators. Similarly, the variation of $0.1 \mathrm{~mm}$ in thickness of the bimorph piezoelectric actuators can cause a shift of about $9 \mathrm{~Hz}$ in resonant frequency. From the above analysis, in the operation of the dithered RLG, the thickness $h_{p}$ of the bimorph piezoelectric actuators is another important factor which influences the resonant frequency beside the spoke. Therefore, the inertia and stiffness induced by the bimorph piezoelectric actuators cannot be neglected. The effect of geometrical parameters for different thickness of the bimorph piezoelectric actuators will be studied in the follow.

The resonant frequency versus the width $b_{p}$ for different thickness of the bimorph piezoelectric actuators is shown in Fig. 9. It is seen that with increase of the width in the range $0.5 \mathrm{~mm}-5.5 \mathrm{~mm}$, the 


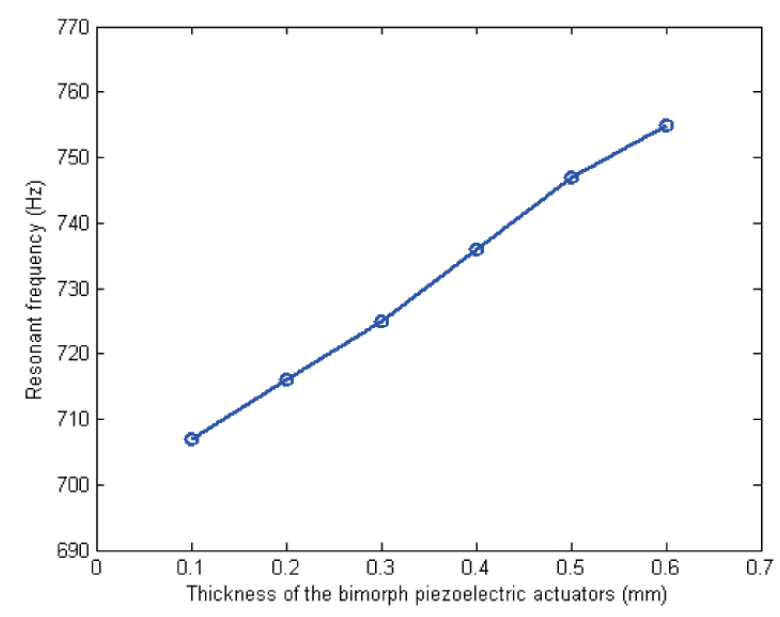

Fig. 8. The relationship between the resonant frequency and thickness of the bimorph piezoelectric actuators.

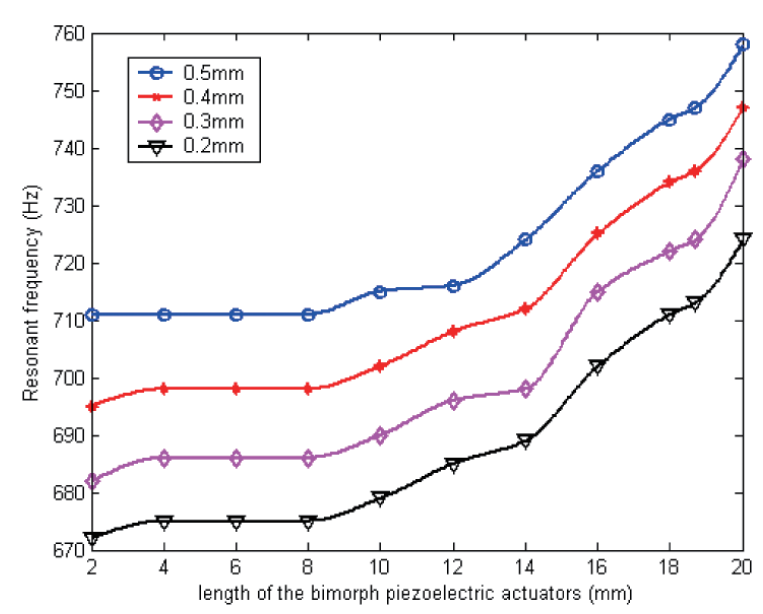

Fig. 10. The relationship between the resonant frequency and length for different thickness of the bimorph piezoelectric actuators.

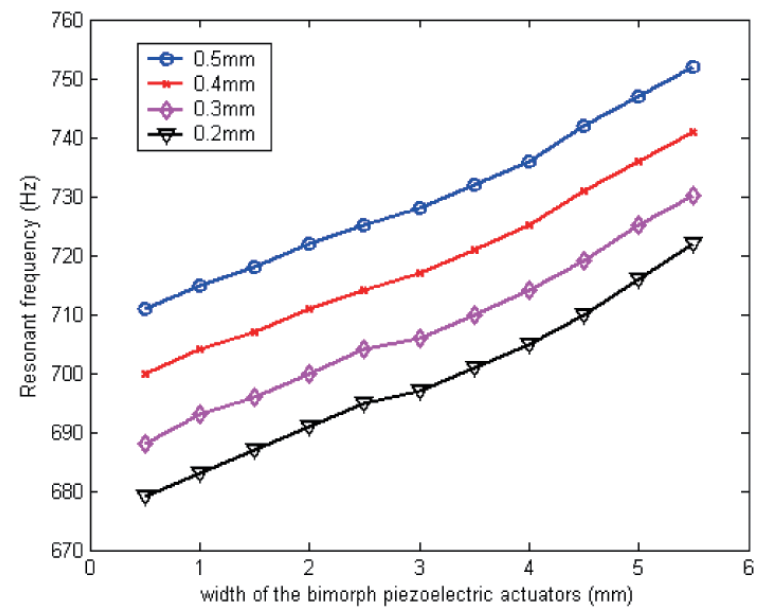

Fig. 9. The relationship between the resonant frequency and width for different thickness of the bimorph piezoelectric actuators.

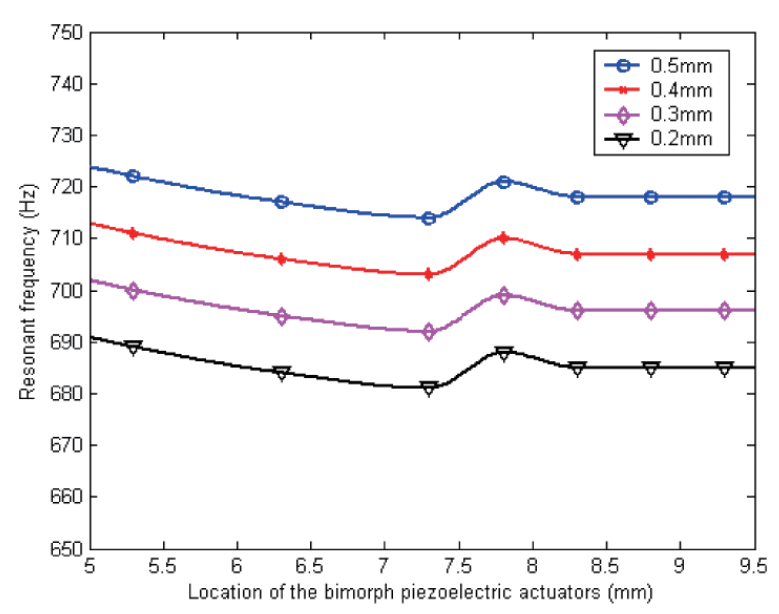

Fig. 11. The relationship between the resonant frequency and location for different thickness of the bimorph piezoelectric actuators.

resonant frequency is increased linearly for different thickness of the bimorph piezoelectric actuators. To analyze the effect of the length $l_{p}$, the resonant frequency is determined while varying $l_{p}$ between $2 \mathrm{~mm}$ to $20 \mathrm{~mm}$ for different thickness. The results are plotted in Fig. 10. The resonant frequency of the dithered RLG presents a non-linger variation with the changes in the length of the bimorph piezoelectric actuators for a given thickness. The influence of location $l_{c}$ of the bimorph piezoelectric actuators from $5 \mathrm{~mm}$ to $9.5 \mathrm{~mm}$ on the resonant frequency for the thickness varying from $0.2 \mathrm{~mm}$ to $0.5 \mathrm{~mm}$ is shown in Fig. 11. The location of the bimorph piezoelectric actuators has a little effect on the resonant frequency which is a helpful guidance for manufacture and installation of the bimorph piezoelectric actuators. From the above analysis we can see that the resonant frequency can be manipulated easily by changing dimension of bimorph piezoelectric actuators. 


\section{Conclusions}

A novel design method based on FEM and experiment is proposed for mechanical dither design in dithered RLG. The resonant frequency of the dithered RLG is stimulated by finite element method. The analysis results show that the resonant frequency depends not only on the geometrical dimensions of the spoke but also on the bimorph piezoelectric actuators. These studies have made it possible to develop mechanical dither of several designs for various applications.

\section{References}

[1] D.H. Titterton and J.L. Weston, Strapdown inertial navigation technology, Institution of Electrical Engineers, (2004).

[2] T. Tucker and E. Levison, The AN/WSN-7B marine gyrocompass/navigator, Institute of Navigation National Technical Meeting (2000), 348-357.

[3] G. Gilster, High accuracy performance capabilities of the military standard ring laser gyro inertial navigation unit, IEEE Position Location and Navigation Symposium (1994), 464-473.

[4] A. Lawrence, Modern inertial technology: Navigation, guidance, and control, Springer-Verlag, Inc, 1998.

[5] S.M. Yang and J.S. Chiu, Dither-motor design with concurrent sensing and actuating piezoelectric materials (Ring laser gyroscope), Smart Mater Struct 3 (1994), 248-253.

[6] J.I. Lahham and J.R. Brazell, Acoustic noise reduction in the MK49 ship's inertial navigation system (SINS), IEEE Position Location and Navigation Symposium (1992), 23-28.

[7] J.I. Lahham, D.J. Wigent and A.L. Coleman, Tuned support structure for structure-borne noise reduction of inertial navigator with dithered ring laser gyros (RLG), IEEE Position Location and Navigation Symposium (2000), 419-428.

[8] B.R. Shackleton, Mechanical design considerations for a ring laser gyro dither mechanism, International Conference on the Mechanical Technology of Inertial Devices (1987), 105-112.

[9] D.C. Lee, G. Moon and J.C. Lee, Mechanical dither design for ring laser gyroscope, KSME International Journal 16 (2002), 485-491.

[10] D.C. Lee, D.W. Yun and C.S. Han, A material rigidity effect of a bimorph piezoelectric actuator, Smart Mater Struct 16 (2007), 1043-1049.

[11] D.C. Lee, J.H. Jang and C.S. Han, Parametric design consideration of a vibro-elastic bimorph piezoelectric converter for a ring laser gyroscope, Smart Mater Struct 15 (2006), 1165-1171.

[12] D.C. Lee, D.I. Shin, Y.S. Kim, J.Y. Lee et al., Modelling the mechanical dither of a ring resonator, Proceedings of the Institution of Mechanical Engineers, Part C: Journal of Mechanical Engineering Science 224 (2010), 1185-1191.

[13] X.D. Yu, G. Wei, X.W. Long et al., Finite element analysis and optimization of dither mechanism in dithered ring laser gyroscope, Int J Precis Eng Manuf 14 (2013), 415-421.

[14] C.H. Nguyen and S.J. Pietrzko, FE anaysis of a PZT-actuated adaptive beam with vibration damping using a parallel R-L shunt circuit, Finite Elem Anal Des 42 (2006), 1231-1239.

[15] Q.P. Wang, X.B. Pei, Q. Wang et al., Finite element analysis of a unimorph cantilever for piezoelectric energy harvesting, Int J Appl Electrom 40 (2012), 341-351.

[16] D.L. Devoe and A.P. Pisano, Modeling and optimal design of piezoelectric cantilever microactuators, J Microelectromech Syst 6 (1997), 266-270.

[17] Q. Li, M. Lovell, J.F. Mei et al., A Study of displacement distribution in a piezoelectric heterogeneous bimorph, J Mech Des 126 (2004), 757-762.

[18] R.R. Craig, Structural dynamics: An introduction to computer methods, Wiley, 1981. 